\title{
Männer fahren LKW, Frauen erziehen Kinder
}

Facebook spielt Stellenanzeigen in diskriminierender Weise an die Nutzer:innen seiner Plattformen aus. Untersuchungen von AlgorithmWatch zeigen, dass sich Facebook an groben Genderstereotypen zu orientieren scheint, um die Zielgruppe von Anzeigen zu bestimmen - auch wenn die Auftraggeber:innen die Ausschreibungen genderneutral gestalten. Die Untersuchungen von AlgorithmWatch machen deutlich, dass das klassische Antidiskriminierungsrecht, insbesondere das AGG in seiner aktuellen Form, den Herausforderungen einer sogenannten „Algorithmendiskriminierung“ nicht gewachsen ist. Insbesondere die mangelnden Sanktionsmöglichkeiten von Diskriminierungen ohne identifizierbares Opfer, werden mit dem zunehmenden Einsatz algorithmischer Systeme mehr und mehr zum Problem.

\section{Das Experiment von AlgorithmWatch}

AlgorithmWatch hat unter anderem auf Facebook Werbeanzeigen für unterschiedliche Jobs geschaltet. Das zweistufige Experiment führte die NGO in mehreren europäischen Ländern durch, unter anderem in Deutschland. Im ersten Schritt hat AlgorithmWatch für Stellenausschreibungen unter anderem als LKW-Fahrer:in und Erzieher:in geworben. Die Ausschreibungen waren stets im Maskulinum formuliert („LKW-Fahrer“, „Erzieher“) und enthielten je ein Symbolbild, das zur beworbenen Tätigkeit passte. Die Werbeanzeige auf Facebook war mit tatsächlichen Ausschreibungen auf Indeed verlinkt, einer Suchmaschine für Stellenangebote. Über die Eingrenzung der angestrebten geographischen Reichweite hinaus hat AlgorithmWatch kein Targeting durchgeführt, also nicht weiter eingegrenzt, wer die Anzeige auf Facebook sehen soll. Danach hat AlgorithmWatch beobachtet, an welche Nutzer:innen Facebook die Anzeigen ausgespielt hat. Das Ergebnis offenbart einen krassen Genderbias. So wurde in Deutschland die Anzeige für eine Stelle als „LKW-Fahrer“ an 4.864 Männern (92,65 \%) ausgespielt, aber nur an 386 Frauen (7,35 \%). Die parallel geschaltete Anzeige für einen Job als „Erzieher" wurde dagegen nur 258 Männern (3,84 \%), aber 6.456 Frauen $(96,16 \%)$ angezeigt.

\section{Nach welchen Kriterien spielt Facebook die Anzeigen aus?}

Facebook verspricht Werbeanzeigen an die Nutzer:innen auszuspielen, für die sie am relevantesten sind. Wenn - wie hier - die werbende Stelle die Zielgruppe der Anzeige nicht näher bestimmt, nimmt also der Facebook-Algorithmus eigenes Targeting vor. Wie Facebook für das Targeting ermittelt, welche Anzeige für welche Personen „relevant“ ist, verrät Facebook nicht. 
Um herauszubekommen, wie der beobachtete Genderbias zustande kam, hat AlgorithmWatch in Frankreich ein zweites Experiment gestartet, diesmal aber Bild und Anzeigentext variiert. Den Ausgangspunkt bildete die im Maskulinum formulierte Anzeige („chauffeur"), die mit dem Foto eines LKW bebildert war. Nur 15 $\%$ der Personen, an die diese Anzeige ausgespielt wurde, waren Frauen. Dagegen erreichte die gleiche Anzeige, die ebenfalls im Maskulinum formuliert, aber mit Kosmetikartikeln bebildert war, zu 88 \% Frauen. Eine Anzeige wiederum, die im Femininum formuliert für eine Stelle als „LKW-Fahrerin“ („chauffeuse“) warb und mit einem LKW bebildert war, wurde nur zu $19 \%$ an Frauen ausgespielt. Auch eine genderneutral formulierte Anzeige („LKW-Fahrer:in“, „chauffeur:se“) mit dem Bild eines LKW erreichte nur zu 22 \% Frauen. Aus den Ergebnissen der Studie schließt AlgorithmWatch, dass der Facebook-Algorithmus hauptsächlich anhand der Bilder entscheidet, wer welche Anzeige zu sehen bekommt - und dabei Genderstereotype bedient.

Dass der offenbarte Genderbias aus gesellschaftlicher Perspektive problematisch ist, liegt auf der Hand. Wenn Stellenausschreibungen für LKW-Fahrer:innen ausschließlich auf Facebook geschaltet würden, hätten Frauen kaum eine Chance, sich auf die Stelle bewerben zu können. Oder - wie im ersten Experiment - hätten Männer kaum eine Chance von der Stelle als „Erzieher:in“ zu erfahren. Indem der Facebook-Algorithmus Anzeigen anhand stereotyper Geschlechterbilder ausspielt, werden Personen nach Geschlecht von bestimmten Jobs und Lebensbereichen ferngehalten. Auf diese Weise werden gesellschaftliche Ungleichheiten perpetuiert und strukturelle Diskriminierungen in die Zukunft verlängert.

\section{Antidiskriminierungsrechtliche Grenzen zulässiger Zielgruppenorientierung}

Aus rechtlicher Perspektive zeigt das Experiment einmal mehr, dass das Phänomen sogenannter Algorithmendiskriminierung schwer greifbar ist. Insbesondere wird deutlich, dass das AGG unzureichend ausgestattet ist, um das grundgesetzlich und unionsrechtlich vorgegebene Ziel zu erreichen, die (tatsächliche) Gleichbehandlung von Personen aller Geschlechter umzusetzen.

Zwar ist nachvollziehbar, dass Facebook ebenso wie Werbende ein Interesse daran haben, dass Anzeigen nur bzw. überwiegend ganz bestimmte Personengruppen ansprechen. In vielen Fällen ist es auch legitim, Stellenanzeigen gezielt an bestimmte Personenkreise auszuspielen. Das ist beispielsweise der Fall, wenn ein Job eine Qualifikation voraussetzt. Wenn die Ausschreibung einer Stelle als Chirurg:in nur an Personen gerichtet ist, die Medizin studiert haben, wird niemand etwas dagegen haben. Die Zielgruppenorientierung nach sachlichen Kriterien wie der „Qualifikation“ ist in der Regel unproblematisch. Problematisch ist dagegen die Kategorisierung von Menschen anhand (gleichheits-)rechtlich geschützter Merkmale wie dem (zugeschriebenen) Geschlecht. Das ist im Grundsatz grundrechtlich verankert in Art. 3 Abs. 2, Abs. 3 GG und Art. 21 Abs. 1 GrCh. Diese Diskriminierungsverbote werden durch EU-Richtlinien und einfache nationale Gesetze insbesondere für Private ausdifferenziert, in Deutschland maßgeblich durch das AGG.

\section{Der gesamte Ausschreibungsprozess muss neutral sein}


Für den Zugang zu Erwerbstätigkeit erklärt bereits § 2 Abs. 1 Nr. 1 AGG die Benachteiligung von Personen u.a. aufgrund des Geschlechts für unzulässig. Der Grundsatz wird konkretisiert durch § 11 AGG, der das Neutralitätsgebot ausdrücklich auf die Ausschreibung eines Arbeitsplatzes erstreckt. Danach muss eine Stellenanzeige nicht nur geschlechtsneutral formuliert sein. Der gesamte Ausschreibungsprozess, also auch die Art, Weise und Reichweite der Bekanntgabe, muss darüber hinaus so erfolgen, dass sie Personen jeden Geschlechts gleichermaßen erreicht. Wird beispielsweise eine genderneutral formulierte Anzeige einer Stelle als „Kellner:in $(\mathrm{m} / \mathrm{w} / \mathrm{d})$ " ausschließlich auf der Damentoilette ausgehangen, haben Männer kaum die Möglichkeit davon zu erfahren, sich zu bewerben und Zugang zu dem Job zu erhalten. Das wäre eine unzulässige Benachteiligung i.S.d. §11 AGG.

Was heißt das nun für die Ausspielung der Stellenanzeigen auf Facebook? Die von AlgorithmWatch genderneutral formulierte Anzeige für eine Stelle als „LKWFahrer:in" wurde nur zu $22 \%$ an Frauen ausgespielt. Die im Maskulinum formulierte Anzeige für einen Job als „Erzieher" erreichte dagegen nur zu 3,84 \% Männer. Werden solchen Anzeigen ausschließlich auf Facebook geschaltet, haben Frauen nur eine sehr geringe Möglichkeit, sich auf die Stelle als LKW-Fahrer bewerben zu können. Umgekehrt haben Männer nur eine minimale Chance, auf die ErzieherStelle aufmerksam zu werden und „Zugang zu der Erwerbstätigkeit“ zu erhalten. Ein Verstoß gegen § 11 AGG liegt insoweit also vor.

\section{Neutralitätspflicht gilt auch für Dritte}

Dafür ist unerheblich, dass das ausschreibende Unternehmen, hier AlgorithmWatch, den angestrebten Personenkreis gar nicht selbst eingegrenzt hat. Das Targeting erfolgte automatisiert durch Facebook bzw. Facebooks Algorithmus. Das Gebot des $\S 11$ AGG, einen Arbeitsplatz nicht unter Verstoß gegen das Benachteiligungsverbot ausschreiben, richtet sich aber nicht nur an die Arbeitgebenden, sondern an jede Stelle, die am Ausschreibungsprozess beteiligt ist. Das folgt schon aus dem passiv formulierten Wortlaut des § 11 AGG ("Ein Arbeitsplatz darf nicht unter Verstoß gegen $\S 7$ Abs. 1 ausgeschrieben werden.") und ist auch von der Rechtsprechung anerkannt.

\section{Unmittelbares wie mittelbares Gendertargeting ist rechtfertigungsbedürftig}

Gegen eine Verletzung des Neutralitätsgebots ließe sich im Übrigen auch nicht einwenden, dass die Anzeige - wie von Facebook versprochen - den Nutzer:innen nach deren Interesse ausgespielt wurde. Wie AlgorithmWatch herausgefunden hat, entscheidet Facebook hauptsächlich anhand der Bebilderung, wer welche Anzeige zu sehen bekommt. Dass der Facebook-Algorithmus Anzeigen mit LKWBildern hauptsächlich an Männer und mit Kosmetikartikeln bebilderte Anzeigen fast ausschließlich an Frauen ausspielt, kann unterschiedliche Gründe haben.

Denkbar ist zum einen, dass Facebook unmittelbares Gendertargeting betreibt und gezielt an das Geschlecht der Nutzer:innen anknüpft; also LKW-Bilder an Personen ausspielt, die in ihrem Profil das entsprechende Kästchen "Mann“ angekreuzt haben und Fotos von Kosmetikartikeln an „Frauen“. Bei einer solchen unmittelbaren 
Anknüpfung an das Geschlecht läge eine unmittelbare Benachteiligung vor, $\S 3$ Abs. 1 AGG. Unmittelbares Gendertargeting auf dem Arbeitsmarkt ist allenfalls gerechtfertigt, wenn das Geschlecht nach § 8 Abs. 1 AGG ,wegen der Art der auszuübenden Tätigkeit oder der Bedingungen ihrer Ausübung eine wesentliche und entscheidende berufliche Anforderung darstellt". Das ist bei LKW-Fahrer:innen ebenso wenig der Fall wie bei Erzieher:innen. Noch weniger wird durch das hier offenbarte Gendertargeting ein bestehender Nachteil i.S.d. § 5 AGG ausgeglichen, sodass auch diese Rechtfertigungsmöglichkeit ausscheidet.

Denkbar ist aber auch, dass Facebook mittelbares Gendertargeting betreibt und sich - vermeintlich geschlechtsneutral - an den Interessen der Nutzer:innen orientiert. Die Anzeige mit dem LKW-Bild könnte beispielsweise solchen Personen angezeigt werden, die auf Facebook selbst Bilder von Autos teilen, in KfZ-Gruppen aktiv sind oder angegeben haben, dass innen das Magazin „Trucker“ gefällt. Der faktische Genderbias wäre dann darauf zurückzuführen, dass die FacebookFangemeinde des Trucker-Magazins überwiegend aus Männern besteht. Frauen würden bei der Ausschreibug der Stelle dann nicht unmittelbar aufgrund ihres Geschlecht, sondern mittelbar benachteiligt. An der rechtliche Bewertung änderte sich damit allerdings zunächst nichts. Denn nach § 3 Abs. 2 AGG sind auch dem Anschein nach neutrale Maßnahmen unzulässig, die Personen wegen des Geschlechts faktisch gegenüber anderen Personen benachteiligen können. Zwar ist der Tatbestand des § 3 Abs. 2 AGG ausgeschlossen, wenn die mittelbare Benachteiligung „,durch ein rechtmäßiges Ziel sachlich gerechtfertigt ist und die Mittel [...] zur Erreichung dieses Ziels angemessen und erforderlich" sind. Das müssten aber die werbende Stelle bzw. Facebook beweisen - und dafür den Algorithmus offenlegen und nachvollziehbar machen, ob hier eine unmittelbare oder mittelbare Anknüpfung an das Geschlecht vorliegt. Bis dahin ist anzunehmen, dass hier nur die allgemeinen Rechtfertigungsmöglichkeiten nach § 5 und $\S 8$ AGG greifen.

\section{Das große Problem der Rechtsdurchsetzung}

Doch auch wenn damit ein klarer Verstoß gegen § 11 AGG durch Facebook vorliegt, ist es praktisch ausgeschlossen, dass ein deutsches Gericht diesen feststellt. Denn Facebook ist hier nicht der (stellenausschreibende) „Arbeitgeber“ i.S.d. AGG. Ein direktes Vorgehen gegen Facebook ist nach dem AGG daher nicht möglich.

Insbesondere geht das Bundesarbeitsgericht aber davon aus, dass ein Verstoß gegen § 11 AGG keine unmittelbare Rechtsfolge hat. Verstöße gegen das Verbot diskriminierenden Stellenausschreibungen können danach nur als Indiz geltend gemacht werden, um einen Verstoß gegen das Benachteiligungsverbot des $\S 7$ Abs. 1 AGG zu beweisen. Eine diskriminierende Stellenausschreibung kann danach nur die Vermutung begründen (22 AGG), dass die spätere Besetzung der Stelle gegen das Benachteiligungsverbot ( $\$ 7$ Abs. 1 AGG) verstieße.

Gerichte, die sich an der Rechtsprechung des BAG orientieren, würden den Verstoß gegen § 11 AGG bei der Ausschreibung der Stelle als LKW-Fahrer:in also überhaupt nur dann thematisieren, wenn bspw. eine Frau, die sich erfolglos auf die Stelle beworben hat, gegen die Ablehnung ihrer Bewerbung vorgeht und von dem einstellenden Unternehmen Entschädigung oder Schadensersatz, § 15 
AGG, verlangt. Das Gendertargeting auf Facebook, könnte die Frau als Indiz dafür anführen, dass sie bei der Einstellungsentscheidung wegen ihres Geschlechts benachteiligt wurde. Das alles könnte sie freilich nur tun, wenn sie auf anderem Wege als über Facebook von der Stellenausschreibung erfährt oder sie eine der wenigen Frauen ist, die die Anzeige zu sehen bekam. Außerdem müsste die klagende Frau von dem Genderbias in der Ausspielung wissen - was kaum vorstellbar ist. Faktisch wird ein Verstoß gegen § 11 AGG durch Facebook also auch auf diesem Weg kaum gerichtlich festgestellt werden.

Theoretisch besteht zwar noch die Möglichkeit, dass der Betriebsrat des ausschreibenden Unternehmens einen Verstoß gegen $\S 11$ AGG vor Gericht bringt. Betriebsräte können nach $\S 17$ Abs. 2 S. 1 AGG, § 23 Abs. 3 S. 1 BetrVG den:die Arbeitgeber:in bei „groben Verstößen“ gegen § 11 AGG auf Unterlassen in Anspruch nehmen. Dafür müsste ein Betriebsrat aber überhaupt existieren. Voraussetzung ist zudem auch hier, dass der Betriebsrat von dem Gendertargeting durch Facebook erfährt - was auch hier kaum vorstellbar ist.

\section{Nicht nur unbefriedigend, auch rechtlich bedenklich}

Nach den Maßstäben der Rechtsprechung könnte das diskriminierende Gendertargeting durch Facebook vor Gericht also allenfalls im Rahmen individueller Entschädigungs- oder Schadensersatzklagen abgelehnter Bewerber:innen gegen den:die Arbeitgeber:in geltend gemacht werden - und auch das nur theoretisch. Faktisch wird weder ein:e unterlegene:r Bewerber:in noch ein Betriebsrat von dem Genderbias erfahren. Wenn sich kein identifizierbares Opfer findet, das den Gang vor Gericht auf sich nehmen will oder es keinen Betriebsrat gibt bzw. dieser nicht tätig wird, fehlt ein Rechtsschutz sogar nicht nur faktisch, sondern auch in der Theorie. Im Ergebnis bedeutet dies, dass die diskriminierende Ausspielung der Stellenwerbung durch Facebook zwar gegen das AGG verstoßen könnte, dieser Verstoß aber kaum justiziabel ist.

Das ist nicht nur extrem unbefriedigend, sondern auch aus unionsrechtlicher Sicht höchst problematisch. Denn wie der EUGH festgestellt hat, verpflichtet das EU-Recht (Art. 15 RL 2000/43/EG, Art. 17 RL 2000/78/EG und Art. 25 RL 2006/54/EG) die Mitgliedstaaten, Verstöße gegen Diskriminierungsverbote durch „wirksame, verhältnismäßige und abschreckende“ Maßnahmen unabhängig davon zu sanktionieren, ob einzelne Personen durch den Verstoß beschwert sind und individuellen Rechtsschutz als konkrete Opfer einer Diskriminierung in Anspruch nehmen können. Wie die Autor:innen der Evaluation des AGG schon 2016 betonen, fordert der EUGH in dieser Entscheidung zwar nicht ausdrücklich ein Verbandsklagerecht für Antidiskriminierungsverbände. „Für eine ,wirksame, verhältnismäßige und abschreckende' Sanktionierung auch der ,opferlosen“ Diskriminierung, die das Unionsrecht allerdings sehr wohl vorsieht, liegt indessen die Schaffung eines solchen Verbandsklagerechts im AGG besonders nahe."

\section{Typisch für Algorithmendiskriminierung}

Das gilt heute mehr denn je. Denn mit dem zunehmenden Einsatz algorithmischer Systeme in unterschiedlichsten Lebensbereichen nehmen auch die Fälle 
sogenannter „opferlosen“ Diskriminierungen zu. In der Vergangenheit haben gemeinnützige Organisationen, Journalist:innen und Wissenschaftler:innen etliche Diskriminierungen und Diskriminierungsrisiken im Zusammenhang mit Algorithmen aufgedeckt. Teils waren (noch) keine konkreten Personen beschwert, sodass diese auch keinen individuellen Rechtsschutz hätten in Anspruch nehmen können. Doch auch wenn im Zusammenhang mit dem Einsatz algorithmischer Systeme konkrete Personen diskriminiert werden, erfahren diese oftmals nicht von der Benachteiligungen oder können sie jedenfalls nicht darlegen bzw. beweisen.

(cc) EY-SA $^{-S^{2}}$ 